\title{
Rovibrational controlled-NOT gates using optimized stimulated Raman adiabatic passage techniques and optimal control theory
}

\author{
D. Sugny, ${ }^{1}$ L. Bomble ${ }^{2}$ T. Ribeyre, ${ }^{2}$ O. Dulieu, ${ }^{3}$ and M. Desouter-Lecomte ${ }^{2,4, *}$ \\ ${ }^{1}$ Institut Carnot de Bourgogne, UMR 5209 CNRS, Université de Bourgogne, BP 47870, 21078 Dijon Cedex, France \\ ${ }^{2}$ Laboratoire de Chimie Physique, Université de Paris-Sud, Unité Mixte de Recherches 8000, Orsay F-91405, France \\ ${ }^{3}$ Laboratoire Aimé Cotton, CNRS, Université Paris-Sud, Bât. 505, 91405 Orsay Cedex, France \\ ${ }^{4}$ Département de Chimie, B6c, Université de Liège, Sart-Tilman, B-4000 Liège 1, Belgium
}

(Received 31 March 2009; published 23 October 2009)

\begin{abstract}
Implementation of quantum controlled-NOT (CNOT) gates in realistic molecular systems is studied using stimulated Raman adiabatic passage (STIRAP) techniques optimized in the time domain by genetic algorithms or coupled with optimal control theory. In the first case, with an adiabatic solution (a series of STIRAP processes) as starting point, we optimize in the time domain different parameters of the pulses to obtain a high fidelity in two realistic cases under consideration. A two-qubit CNOT gate constructed from different assignments in rovibrational states is considered in diatomic $(\mathrm{NaCs})$ or polyatomic $\left(\mathrm{SCCl}_{2}\right)$ molecules. The difficulty of encoding logical states in pure rotational states with STIRAP processes is illustrated. In such circumstances, the gate can be implemented by optimal control theory and the STIRAP sequence can then be used as an interesting trial field. We discuss the relative merits of the two methods for rovibrational computing (structure of the control field, duration of the control, and efficiency of the optimization).
\end{abstract}

DOI: $10.1103 /$ PhysRevA.80.042325

PACS number(s): 03.67.Lx, 82.50.Nd, 03.67.Ac

\section{INTRODUCTION}

Implementing logical gates on molecular systems for classical Boolean logic $[1,2]$ or quantum computing (see Refs. [3-10] to cite a few) is now a well-recognized topic with numerous theoretical studies and potential experimental applications $[2,11]$. From the theoretical point of view, a large amount of works using optimal control theory (OCT) $[12,13]$ or genetic algorithms (GAs) [14] has been done in the recent literature to implement one- or two-qubit gates using vibrational modes of molecules [3,6,7,15-19] or rotational states $[8,20]$. Recently, even more complex schemes such as a full adder [21], adder-subtractor [22], Deutsch-Jozsa $[3,11,18,20,23,24]$, or Shor [7] algorithms have been considered. Adiabatic passage techniques (APTs) are also powerful methods to transfer population or to implement quantum gates in molecular systems [25]. One of the most wellknown adiabatic techniques is the stimulated Raman adiabatic passage (STIRAP) scheme. For optical cavity, it has been shown that a series of STIRAP processes can be used to implement quantum gates [26,27] and algorithms as, for instance, the Grover's search algorithm [28]. Such systems are ideal in the sense that all the levels populated during the control are degenerate. This is no longer the case for molecular systems where the duration of the control has to be adjusted since the levels are not degenerate. This means that the relative phase of the different states of the gate varies as a function of the total duration of the control.

We consider a two-qubit controlled-NOT (CNOT) gate (controlled-NOT gate in which the target qubit flips if and only if the control qubit is equal to 1) and two particular control techniques: the APT process optimized by a genetic algorithm in time domain and APT coupled to an optimiza-

\footnotetext{
*mdesoute@1cp.u-psud.fr
}

tion by OCT. We show that the efficiency of the transformation driven by APT strongly depends on the assignments of the qubit states to various choices of vibrational and rotational levels of the molecule. A previous study [8] has already examined the fidelity of a CNOT gate according to the choice of the control qubit in a diatomic molecule. This work used shaped mid-infrared pulses optimized by a genetic algorithm to manipulate the qubit states. In this paper, the APT method is based on transitions via an excited electronic state. Therefore, we revisit this problem by operating in the uvvisible spectrum. This has been recently proposed [29] to implement gates from rovibrational states and illustrated in the $\mathrm{Na}_{2}$ molecule. Due to the complexity of the considered systems, the APT process alone does not give a sufficient fidelity. To overcome this difficulty, the idea consists in combining APT and optimization. In a first step, the adiabatic technique allows us to determine the structure of the control field. In a second step, we optimize different parameters to recover a high efficiency for the control. We have chosen an optimization by GA in the time domain, the parameters being the intensity of the different pulses, their duration, and the position of the center of each pulse (these parameters modify the relative positions of the different pulses). For two-qubit gates, the number of parameters is of the order of 10 . Note that such techniques combining adiabatic passage and optimization (without GA) of a finite number of parameters have already been used for the transfer of population in molecules $[16,17,30,31]$. The present approach is thus different from the usual optimization by GA in the frequency domain $[7,8,32]$. When the APT sequence cannot drive the gate transformation, we use OCT with spectral constraints in order to get realistic pulses. The APT sequence constitutes then an interesting trial field to guide the optimization toward a simpler control field even if a part of the robustness of the adiabatic techniques is lost.

We consider two molecular systems: a polar diatomic molecule $\mathrm{NaCs}$ and a polyatomic molecular system $\mathrm{SCCl}_{2}$. 
Polar diatomic molecules in the context of cold molecules [33-35] have been suggested as promising materials for scalable quantum computing $[9,10,36,37]$. The $\mathrm{SCCl}_{2}$ molecule has been already used to simulate quantum computing with excited rovibrational states [7,22].

The paper is organized as follows. In Sec. II, we recall the principles of the adiabatic passage techniques, genetic algorithms, and optimal control theory. The different qubit assignments in rovibrational states are presented in Sec. III. We illustrate the role of the assignment of qubit states in a diatomic system in Sec. IV and in a polyatomic molecule in Sec. V. Conclusions and prospective views are given in Sec. VI. Some technical points are reported in Appendixes A and B.

\section{METHODOLOGY}

The Hamiltonian of each diatomic or polyatomic system is expressed in a rovibrational basis set belonging to two electronic states for which the Born-Oppenheimer approximation is valid. After integration over the electronic coordinates, the Hamiltonian operator acting in the rovibrational nuclear wave-function space reads

$$
\mathbf{H}=\left(\begin{array}{cc}
\mathbf{H}_{0}^{1} & 0 \\
0 & \mathbf{H}_{0}^{2}
\end{array}\right)-\left(\begin{array}{cc}
0 & \boldsymbol{\mu}_{12} \\
\boldsymbol{\mu}_{21} & 0
\end{array}\right) E(t),
$$

where $\mathbf{H}_{0}^{\alpha}=T_{N}+V_{\alpha}(\alpha=1,2)$ is the Hamiltonian describing the nuclear dynamics in the ground $(\alpha=1)$ and excited $(\alpha=2)$ states $\left(T_{N}\right.$ is the kinetic-energy operator of the nuclei and $V_{\alpha}$ is the BO electronic potential energy). $\boldsymbol{\mu}_{12}$ is the electronic transition dipole momentum and $E(t)$ the electric field. In a rovibrational basis set (i.e., after integrating the Schrödinger equation over the nuclear coordinates), each $\mathbf{H}_{0}^{\alpha}$ block is diagonal and contains the rovibrational energies for each electronic state. The off-diagonal blocks $\boldsymbol{\mu}_{\alpha \beta}$ contain all coupling elements. They are discussed in Appendix A. The coupled equations are integrated by the Runge-Kutta method [38] in the interaction representation (but without any rotating-wave approximation). $E(t)$ is chosen linearly polarized so that the simulations are performed for a constant value of the quantum number $M$ (here, $M=0$ ), where $M$ is the projection of the total angular momentum on the polarization direction of the control field which corresponds to the axis $\mathrm{OZ}$ of the laboratory frame (see Appendix A).

\section{A. Description of the adiabatic processes}

We consider an adiabatic process introduced in Refs. $[26,27]$ for optical cavity and applied to molecular systems in Ref. [29]. This process is composed of a series of STIRAP processes [25]. A STIRAP process transfers the population from an initial state to a final state (uncoupled to the initial state) via an intermediate state $|I\rangle$. Transition between the initial state and the intermediate one is called pump and the one between the intermediate and the final state is called Stokes. When the pump-Stokes sequence is applied in a counterintuitive order (the Stokes pulse before the pump pulse) with a properly chosen overlap between the two pulses, the intermediate state $|I\rangle$ is not populated during the

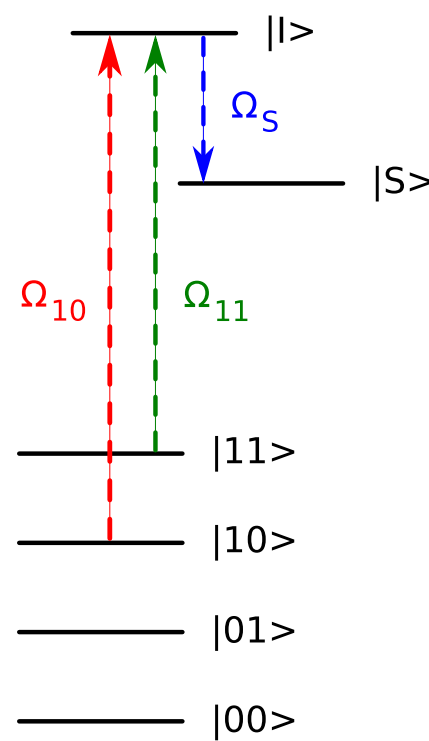

FIG. 1. (Color online) Transitions involved in the adiabatic process for computing a CNOT gate by the pulse sequence $\Omega_{S} \Omega_{10} \Omega_{11} \Omega_{S}$.

transfer. In molecular systems, due to the coupling to other rovibrational states, the state $|I\rangle$ can be slightly populated during the process. For a CNOT gate, the following transitions must be driven by a single control field:

$$
|00\rangle \rightarrow|00\rangle ;|01\rangle \rightarrow|01\rangle ;|10\rangle \rightarrow|11\rangle ;|11\rangle \rightarrow|10\rangle .
$$

The sequence of population inversions proposed in Ref. [29] to implement a CNOT gate by STIRAP techniques is schematized in Fig. 1.

The first STIRAP makes the transfer from $|11\rangle$ to a shelving state $|S\rangle$ by applying $\Omega_{S}$ (Stokes) and then $\Omega_{11}$ (pump) where $\Omega=\mu_{i j} A(t) / \hbar$ is the Rabi frequency for the transition and $\mu_{i j}$ is the dipolar matrix element between the chosen rovibrational states and $A(t)$ is the pulse envelope. The second STIRAP makes the transfer from $|10\rangle$ to $|11\rangle$ by applying $\Omega_{11}$ and then $\Omega_{10}$. The third STIRAP makes the transfer from $|S\rangle$ to $|10\rangle$ by applying $\Omega_{10}$ and then $\Omega_{S}$. All the transfers are realized via $|I\rangle$ which remains slightly populated. By this way, one inverses $|10\rangle$ and $|11\rangle$ leaving $|00\rangle$ and $|01\rangle$ unchanged. In this pulse sequence, when two consecutive pulses are identical, one could use only one pulse instead of two [29]. In that way, a pulse corresponds to the pump for one STIRAP process and to the Stokes pulse for the next STIRAP process. This is very interesting since the optical resources are reduced. The sequence of pulses for a CNOT gate is then $\Omega_{S} \Omega_{11} \Omega_{10} \Omega_{S}$ where the second and fourth pulses have a phase $\exp (i \pi)$ to enforce the correct relative phases of the final states [29]. Adjusting the parameters of the different pulses of the STIRAP processes will be realized by genetic algorithms. The optimization must allow us to realize the four transformations of the CNOT gate with a phase constraint $\frac{1}{2}(|00\rangle+|01\rangle+|10\rangle+|11\rangle) \rightarrow \frac{1}{2}(|00\rangle+|01\rangle+|10\rangle+|11\rangle) e^{i \varphi}$,

where the phase $\varphi$ can take any value between 0 and $2 \pi$. This transformation ensures that the phases of the final states 
of the gate are equal and that the pulse can realize the gate transformation on any superposition of the computational basis states $[3,39]$. In other words, this implies that a true unitary transformation is implemented by the control field. If the phase constraint is not satisfied, then only the population inversion is realized. This means therefore that a classical and not a quantum gate is implemented by the laser fields. In the different numerical computations, we will see that the transformation on the phase is the most difficult to realize. The simulations are done in the interaction representation (which gives a good numerical stability) so that the dynamical phases are omitted (see Ref. [29]). However, the time scale of the rovibrational field-free dynamics is of the order of $100 \mathrm{ps}$ and the phase constraint in the Schrödinger representation is expected to be satisfied after a similar timing which is shorter than the duration of the pulse.

\section{B. Optimization by genetic algorithms}

We describe in this section the computational details of GA we have used [40]. We consider a series of Gaussian pulses which are characterized by their intensity, their duration, and the position of the maximum of the pulse. This last parameter allows us to modify the delay between the different fields. Each Gaussian pulse has the following general form:

$$
E_{k}(t)=E_{0 k} \exp \left[-\left(t-t_{k}\right)^{2} / 2 \gamma_{k}^{2}\right] \cos \left(\omega_{k} t\right) .
$$

The carrier frequency $\omega_{k}$ is fixed to the value of the corresponding transition. The parameters optimized by GA are $E_{0 k}$ and $t_{k}$ for each pulse, $\gamma_{k}$ being fixed. Since the sequence is composed of four pulses, we have eight parameters to optimize. We have used GA with the following characteristics. Each individual is formed by the eight parameters (the chromosomes). At each iteration of the algorithm, we consider a population of 12 individuals and we compute the fitness function corresponding here to the fidelity of the CNOT gate. We choose 12 new individuals by selecting 12 times the best among three drawn randomly (it is possible that the same parent can be chosen several times). This new set of individuals is modified by mutation (the probability of mutation is 0.5 ) or by crossover (the probability of crossover is 0.8 ). The fitness of these new individuals is calculated and the algorithm is iterated. Different choices can be done to estimate the fitness of a gate with the phase constraint $[8,20,41]$. To accelerate the convergence of the algorithm and to keep the structure of the STIRAP sequence at each step of the algorithm, we define the fitness not from the final fidelity of the gate but from a mean over the fidelity of the three population inversions involved in the sequence. With this definition, one has to assess the fidelity $F_{n}$ after each STIRAP process $n$ with $n=1,2,3$. This intermediate fidelity $F_{n}$ is defined as the overlap between the time-depending wave function $\psi_{i}^{n}(t)$ (initial wave function evolving because of the field) and the wave function which the transformation drives toward $\phi_{f}^{n}\left(t_{n}\right)$ at the final time of step $n: F_{n}$ $=\left|\left\langle\psi_{i}^{n}(t) \mid \phi_{f}^{n}\left(t_{n}\right)\right\rangle\right|^{2}$. For example, for the first inversion, $n=1$, we have

$$
\begin{aligned}
\psi_{i}^{n}(t=0) & =\frac{1}{2}(|00\rangle+|01\rangle+|10\rangle+|11\rangle) \rightarrow \phi_{f}^{n}\left(t_{n}\right) \\
& =\frac{1}{2}(|00\rangle+|01\rangle+|10\rangle+|S\rangle) .
\end{aligned}
$$

The initial state is a superposition of the qubit states in order to reach the phase constraint [Eq. (2)]. The global fidelity, defined as $F=\left(F_{1}+F_{2}+F_{3}\right) / 3$, is calculated for each individual.

\section{Optimization by optimal control}

The other method used to improve or replace the STIRAP sequence is the multitarget optimal control theory $[12,13]$. The optimal field maximizes the objective functional with the constraint that the Schrödinger equation is satisfied at any time

$$
\begin{aligned}
J= & \sum_{n=1}^{Z}\left\{\left|\left\langle\psi_{i}^{n}\left(t_{f}\right) \mid \phi_{f}^{n}\right\rangle\right|^{2}-2 \operatorname{Re}\left[\int_{0}^{t_{f}}\left\langle\psi_{f}^{n}(t)\left|\partial_{t}+\frac{i}{\hbar} \hat{H}\right| \psi_{i}^{n}(t)\right\rangle d t\right]\right\} \\
& -\alpha \int_{0}^{t_{f}} E^{2}(t) d t
\end{aligned}
$$

where $\alpha$ is a positive penalty factor chosen to weight the importance of the laser fluence. For a $N$-qubit gate, $Z=2^{N}+1$, where $2^{N}$ is the number of input-output transitions in the gate unitary transformations and the supplementary equation is the phase constraint [Eq. (2)]. The $\psi_{i}^{n}(t)$ are the wave packets which are propagated forward in time in interaction representation (as in the APT approach) with the initial conditions $\psi_{i}^{n}(t=0)=\phi_{i}^{n}, n=1, \ldots, Z$. The Lagrange multipliers $\psi_{f}^{n}(t)$ are propagated backward in time with the final conditions $\psi_{f}^{n}\left(t=t_{f}\right)=\phi_{f}^{n}, n=1, \ldots, Z$. Note that for a twoqubit gate, $N=2$ and $Z=2^{2}+1=5$. The universal gate field able to drive any input toward the corresponding output is a sum of $Z$ contributions

$$
E_{j}(t)=-(s(t) / \hbar \alpha) \operatorname{Im}\left[\sum_{n=1}^{Z}\left\langle\psi_{f}^{n}(t)\left|\mu_{j}\right| \psi_{i}^{n}(t)\right\rangle\right],
$$

where $j$ denotes the polarization direction of the electric field. It is chosen to be the OZ laboratory axis. A switching function $s(t)=\sin ^{2}\left(\pi t / t_{f}\right)$ is introduced to provide a smooth on and off switch of the field [4]. At each iteration noted $(k)$, the field is given by $E_{j}^{(k)}=E_{j}^{(k-1)}+\Delta E_{j}^{(k)}$, where $\Delta E_{j}^{(k)}$ is calculated by Eq. (5) [41] and $\mu_{j}$ is expressed in interaction representation. The fidelity of the gate $F$ is given by the average performance index of each transformation including that corresponding to the phase constraint

$$
F=\frac{1}{Z} \sum_{n=1}^{Z}\left|\left\langle\psi_{i}^{n}(t) \mid \phi_{f}^{n}\right\rangle\right|^{2} .
$$

Usually, OCT leads to complicated field containing unrealistic frequencies too low or too high according to the model or to the corresponding experimental process and some filtering is necessary to improve the field. We apply a filtering on the Fourier transform of the optimal field. The filter is composed 
of bandpass filters around the vibrational frequencies of the model with a bandwidth fixed to a fraction of the vibrational frequencies (see below for details). This scheme does not preserve the monotonic convergence of the algorithm but however a good convergence is achieved. Note that monotonically convergent algorithms with filtering have been proposed recently in the literature [42].

\section{QUBIT ASSIGNMENT IN MOLECULAR STATES}

The four logical states involved in the CNOT gate are obtained by varying the value of the controlled and the target qubits $q_{c}=0,1$ and $q_{t}=0,1$ leading to the computational basis set states $|00\rangle,|01\rangle,|10\rangle$, and $|11\rangle$. The CNOT transformation is

$$
U_{\mathrm{CNOT}}=\left(\begin{array}{cccc}
1 & 0 & 0 & 0 \\
0 & 1 & 0 & 0 \\
0 & 0 & 0 & 1 \\
0 & 0 & 1 & 0
\end{array}\right) .
$$

The four logical states can be mapped onto the molecular rovibrational states in different ways. The molecular states are denoted by two quantum numbers $v$ and $J$ related to the vibrational and the rotational degrees of freedom, respectively. (The quantum number $M$ is fixed to $M=0$ ). Among the different assignment possibilities presented below, we first consider two values of $v\left(v_{1}\right.$ and $\left.v_{2}\right)$ and two values of $J$ $\left(J_{1}\right.$ and $\left.J_{2}\right)$ to choose the four molecular states. Even in this way, there are still two possibilities to assign the controlled qubit $q_{c}$.

(1) $q_{c}$ is encoded in the rotation $q_{c}=0 \rightarrow J=J_{1}$ and $q_{c}=1 \rightarrow J=J_{2}$. The target $q_{t}$ is thus in the vibration. The computational basis set is then mapped onto

$$
|00\rangle,|01\rangle,|10\rangle \text {, and }|11\rangle \rightarrow\left|J_{1} v_{1}\right\rangle,\left|J_{1} v_{2}\right\rangle,\left|J_{2} v_{1}\right\rangle \text {, and }\left|J_{2} v_{2}\right\rangle \text {. }
$$

The CNOT transformation leads to a transition between two target vibrational states $v_{1} \leftrightarrow v_{2}$ for the population of the rotational state $J_{2}$ but to no transition for the population of the state $J_{1}$.

(2) $q_{c}$ is encoded in the vibration states $q_{c}=0 \rightarrow v=v_{1}$ and $q_{c}=1 \rightarrow v=v_{2}$. The target $q_{t}$ is now in the rotation. The computational basis set is then mapped onto

$|00\rangle,|01\rangle,|10\rangle$, and $|11\rangle \rightarrow\left|v_{1} J_{1}\right\rangle,\left|v_{1} J_{2}\right\rangle,\left|v_{2} J_{1}\right\rangle$, and $\left|v_{2} J_{2}\right\rangle$.

The CNOT transformation leads to the inversion of the population of two target rotational states only if the vibrational state is $v_{2}$ and no transition occurs if the vibrational state is $v_{1}$.

(3) $q_{c}$ and $q_{t}$ are encoded in four different vibrational states

$|00\rangle,|01\rangle,|10\rangle$, and $|11\rangle \rightarrow\left|v_{1} J_{1}\right\rangle,\left|v_{2} J_{2}\right\rangle,\left|v_{3} J_{3}\right\rangle$, and $\left|v_{4} J_{4}\right\rangle$.

The third possibility consists in choosing different rotational states belonging to four different vibrational states. The interpretation of a controlled qubit in terms of the rotation or of the vibration is lost. The CNOT transformation corresponds to an inversion of population between two rovibrational states

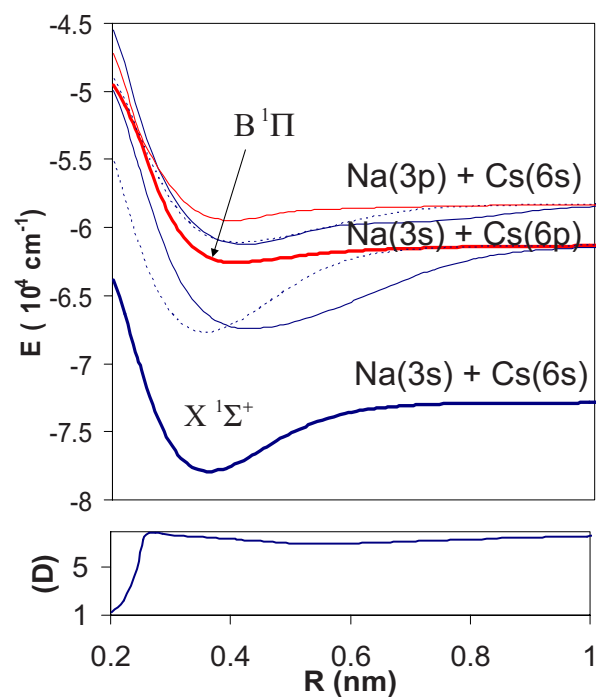

FIG. 2. (Color online) Upper panel: Potential energy curves of NaCs. The ${ }^{1} \Sigma^{+}$and ${ }^{1} \Pi$ singlet states are in full lines (blue for the ${ }^{1} \Sigma^{+}$states and red for the ${ }^{1} \Pi$ states) and the triplet states ${ }^{3} \Pi$ are in dotted lines. The selected electronic states are the ground $X^{1} \Sigma^{+}$and $B{ }^{1} \Pi$ states (noted by an arrow). Lower panel: Electronic transition dipole moment between the selected $X^{1} \Sigma^{+}$and $B{ }^{1} \Pi$ electronic states.

of two different vibrational manifolds $v_{3} \leftrightarrow v_{4}$. The difference with case (1) comes from the fact that the population of a rotational state $J_{1}$ or $J_{2}$ which remains unaltered belong to different vibrational states $v_{1}$ or $v_{2}$.

\section{DIATOMIC SYSTEM}

We now examine the feasibility of the different assignments in rovibrational states $|\alpha, v, J M \Omega\rangle$ of a diatomic molecule. The quantum numbers denote the electronic $(\alpha)$, vibrational $(v)$, and rotational states $(J, M)$, respectively. $\Omega=\Lambda+\Sigma$, where $\Lambda$ is the projection of the total electronic angular momentum on the molecular axis and $\Sigma$ the projection of the total electronic spin. $\Lambda=0$ for the ${ }^{1} \Sigma^{+}$state, $\Lambda=1$ for the ${ }^{1} \Pi$ state, and $\Sigma=0$ for both states. The electronic potential-energy curves of the $\mathrm{NaCs}$ molecule have been computed and published recently [43]. They are shown in Fig. 2. The four qubit states and the shelving state are rovibrational states of the ${ }^{1} \Sigma^{+}$ground electronic state $(\alpha$ $=1$ ). The excited electronic state supporting the intermediate state $|I\rangle$ must not interact non-adiabatically through pronounced avoided crossings generated by the strong spin-orbit interaction between singlet and triplet states. Such interactions should lead to a nonradiative decay of the intermediate state. As seen in Fig. 2, the bottom of the lower $B{ }^{1} \Pi$ state $(\alpha=2)$ is well isolated from the other ones so that no strong interaction is expected. It is thus a good candidate to support the rovibrational intermediate state $|I\rangle$.

The energies of the rovibrational states are estimated by the approximation of the rigid rotor valid for the low levels

$$
E_{\alpha, v, J}=E_{\alpha}^{0}+(v+1 / 2) \hbar \omega_{\alpha}+\left[J(J+1)-\Omega^{2}\right] B_{\alpha}(v) .
$$

The rotational constant for each vibrational state $v$ is given by $B_{\alpha}(v)=\frac{\hbar^{2}}{2 m}\left\langle v\left|\frac{1}{R^{2}}\right| v\right\rangle$, where $R$ is the internuclear distance 


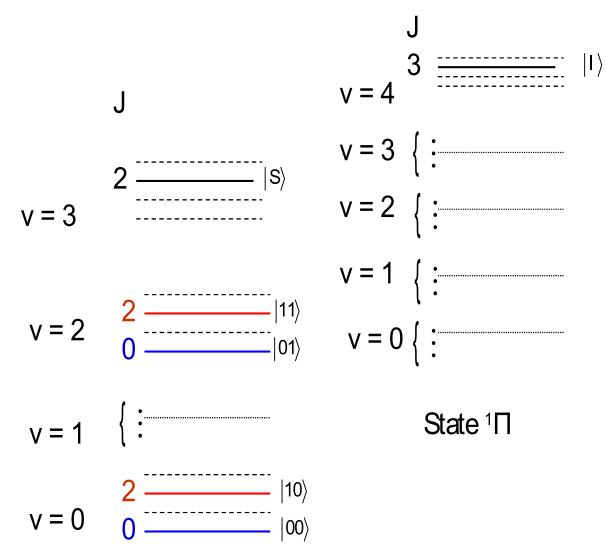

State ${ }^{1} \Sigma^{+}$

FIG. 3. (Color online) Scheme of the qubit states with $q_{c}$ in the rotation for the diatomic molecule. $v$ and $J$ are the vibrational and rotational quantum numbers, respectively. $|S\rangle$ and $|I\rangle$ are the shelving and intermediate states of the STIRAP sequence (see Fig. 1).

and $m$ the reduced mass. The basis set for the numerical simulation contains the rotational states up to $J=5(M=0)$ of the vibrational states $v=0,1,2,3$ in the ${ }^{1} \Sigma^{+}$state. For the ${ }^{1} \Pi$ state, one retains the $v=4$ state and the rotational states from $J=1$ (lowest value for a $\Pi$ state) to $J=4$. The size of basis set is chosen sufficiently large to avoid numerical boundary errors. All the vibrational states involved in the assignment 3 are kept. Other vibrational states are safely discriminated given the pulse duration chosen here. Rotational states are retained by taking into account the $\Delta J= \pm 1$ selection rule. The calculation of the matrix elements of the electronic transition moment $\mu_{12}$ [Eq. (1)] in the rovibrational basis set is summarized in Appendix A.

\section{A. Assignment 1: Control qubit $q_{c}$ encoded in the rotational states}

Figure 3 schematizes the rovibrational states used and gives their quantum numbers (the energy scale is arbitrary).

\section{APT and $G A$}

One observes that the STIRAP sequence is not efficient when the control qubit is encoded in a pure rotational state, for instance, $J=0$ (logical state 0$)$ and $J=2$ (logical state 1 ). The parameters of the pulses giving the largest possible fidelity are gathered in Appendix B. A vibrational transition must occur only from the state $J=2$ and not affect the state $J=0$ belonging to the same vibrational state. As shown in the lower panel of Fig. 4, it is possible to optimize a STIRAP sequence realizing the transformation $|11\rangle$ $\left(\left|{ }^{1} \Sigma^{+}, 2,2\right\rangle\right) \rightarrow|10\rangle\left(\left|{ }^{1} \Sigma^{+}, 0,2\right\rangle\right)$ or vice versa but this solution does not preserve the populations in states $|00\rangle\left(\left|{ }^{1} \Sigma^{+}, 0,0\right\rangle\right)$ and $|01\rangle\left(\left|\Sigma^{1}, 2,0\right\rangle\right)$ as illustrated in the upper panel of Fig. 4.

Figure 5 gives a scheme of the levels and of the corresponding transitions which explains why the $\Omega_{S} \Omega_{11} \Omega_{10} \Omega_{S}$ sequence also interacts with the $|00\rangle$ and $|01\rangle$ states. Due to the regularity of the rotational levels, the energy gaps be-

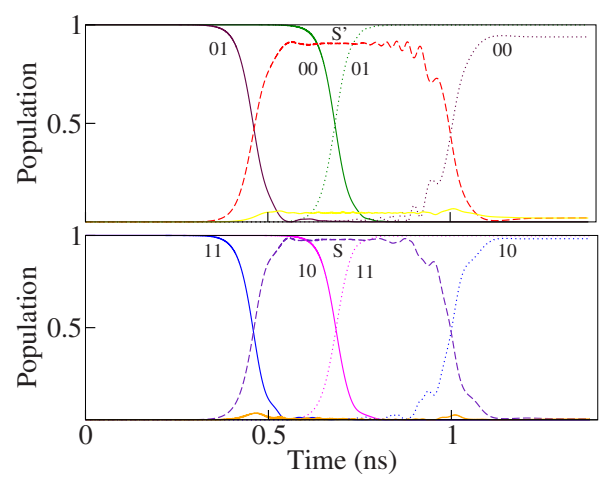

FIG. 4. (Color online) CNOT gate by APT with the control qubit encoded in the rotational states $J=0$ and $J=2$ and the target qubit in the vibrational states $v=0$ and $v=2$ (see Fig. 3). Upper panel: Evolution of the populations which should remain unaltered when the control qubit is $0, \quad|01\rangle \quad$ (full line) $\rightarrow\left|S^{\prime}\right\rangle \quad$ (dashed line) $\rightarrow|00\rangle$ (dotted line) and $|00\rangle$ (full line) $\rightarrow|01\rangle$ (dotted line). The new shelving state found by the system is $\left|S^{\prime}\right\rangle=\left|{ }^{1} \Sigma^{+}, 3,0\right\rangle$ and the new intermediate state is $\left|I^{\prime}\right\rangle=\left|{ }^{1} \Pi, 4,1\right\rangle$. Lower panel: Evolution of the populations of the states when the control qubit is 1 (see Fig. 1), $|11\rangle \quad$ (full line) $\rightarrow|S\rangle$ (dashed line) $\rightarrow|10\rangle$ (dotted line) and $|11\rangle$ (full line) $\rightarrow|10\rangle$ (dotted line).

tween the states $|00\rangle$ and $|01\rangle$ and some states acting as intrusive shelving state $\left.\left|S^{\prime}\right\rangle=\left.\right|^{1} \Sigma^{+}, 3,0\right\rangle$ and intermediate one $\left|I^{\prime}\right\rangle=\left|{ }^{1} \Pi, 4,1\right\rangle$ have the same orders of magnitude as the frequencies of the pulse. Since the STIRAP process allows a detuning between the frequency of the laser field and the transition frequency of the energy levels [25], this leads to unwanted transitions. The same negative result has been obtained for longer duration of pulses of the order of some

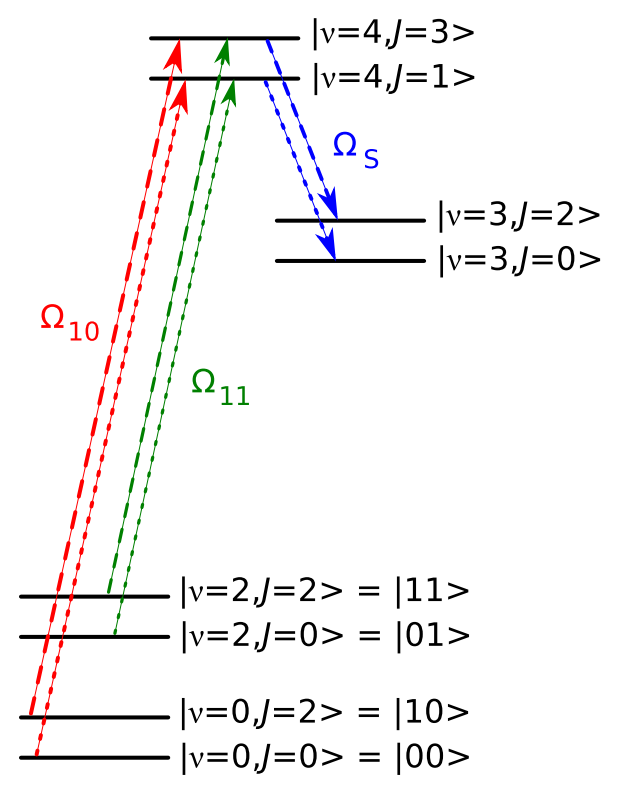

FIG. 5. (Color online) Scheme showing the unwanted transitions due to the $\Omega_{S} \Omega_{11} \Omega_{10} \Omega_{S}$ pulse sequence with assignment 1 (control qubit encoded in the rotational states). The expected transitions from the $|10\rangle$ and $|11\rangle$ states are drawn in dashed lines. The unwanted transitions from the $|00\rangle$ and $|01\rangle$ states are drawn in dotted lines (intrusive shelving state $\left|S^{\prime}\right\rangle=\left|{ }^{1} \Sigma^{+}, 3,0\right\rangle$ and intrusive intermediate one $\left.\left|I^{\prime}\right\rangle=\left|{ }^{1} \Pi, 4,1\right\rangle\right)$. 

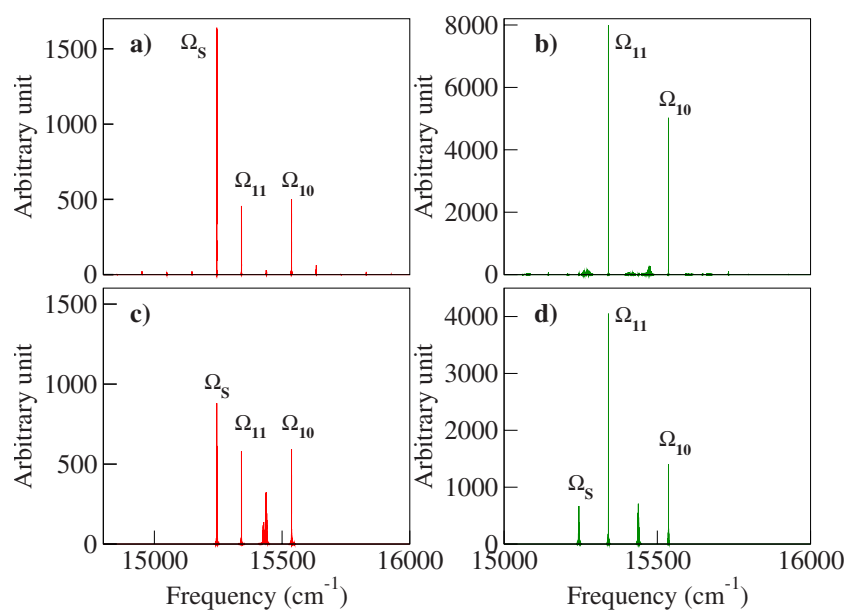

FIG. 6. (Color online) Frequencies in $\mathrm{cm}^{-1}$ of the Fourier transform of the OCT field for the CNOT gate with the control qubit in the rotational states $J=0$ and $J=2$ and the target qubit in the vibrational states $v=0$ and $v=2$ (see Fig. 3) for the NaCs system. (Upper panels) First optimization without filtering (a) with the STIRAP sequence as a trial field and (b) with a guess field containing the frequencies $\Omega_{11}$ and $\Omega_{10}$. (Lower panels) Spectrum of the optimal fields after filtering.

hundreds of nanoseconds. As the population inversion already breaks down, we do not introduce the phase constraint but we further optimize the gate by OCT.

\section{2. $O C T$}

This example illustrates that STIRAP processes are not pertinent to assign the control qubit in pure rotational states. However, this scheme can be used with OCT in a standard multitarget optimal control algorithm. We have optimized the five transformations of the CNOT gate with a phase constraint [Eq. (2)]. We recall that this constraint allows us to implement not only population inversions but also a complete unitary transformation, which is required in quantum computing. OCT permits in principle to find pulses with shorter duration and to work in different spectral range. Here, we consider the uv-visible range by cycling via an excited electronic state. In this case, OCT requires a starting guess containing at least one frequency corresponding to the electronic transition. We compare two trial fields and two pulse durations. We use a first-trial field composed of two Gaussian pulses with carrier frequencies corresponding to $\Omega_{11}$ and $\Omega_{10}$ (see Fig. 1). The parameters of each Gaussian pulse [Eq. (3)] are $\gamma=0.29 \mathrm{~ns}, t=0.69 \mathrm{~ns}$, and $E_{0}=10.2810^{6} \mathrm{~V} \mathrm{~m}^{-1}$. We also try the STIRAP sequence as a trial field with carrier frequencies $\Omega_{11}, \Omega_{10}$, and $\Omega_{S}$. The parameters are gathered in Table A of Appendix B. This choice allows us to guide the algorithm toward a particular mechanism. For each case, we compare a long pulse (1.4 ns) and another pulse 10 times shorter. We first discuss the long duration pulses. We compute the Fourier transform of the optimal fields converged with a fidelity of $99.9 \%$ in about ten iterations. They are shown in the upper panels of Fig. 6. The optimized field before filtration seems very simple. It mainly contains the frequencies of the trial field but also some weak transitions

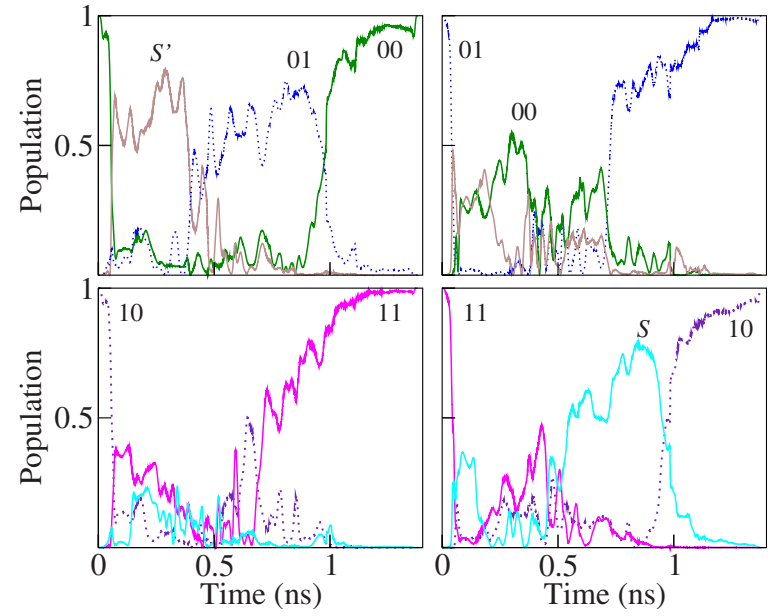

FIG. 7. (Color online) CNOT gate with phase constraint [Eq. (2)] optimized by OCT with the control qubit encoded in the rotational states $J=0$ and $J=2$ and the target qubit in the vibrational ones $v=0$ and $v=2$ (see Fig. 3). Population evolution of the four qubit states: $|00\rangle\left(\left|{ }^{1} \Sigma^{+}, 0,0\right\rangle\right)$ in full lines, $|01\rangle\left(\left|{ }^{1} \Sigma^{+}, 2,0\right\rangle\right)$ in dotted line, $|10\rangle\left(\left|{ }^{1} \Sigma^{+}, 0,2\right\rangle\right)$ in dotted line, and $\left.|11\rangle\left(\left.\right|^{1} \Sigma^{+}, 2,2\right\rangle\right)$ in full line. One observes intermediate populations of the state $\left.\left|S^{\prime}\right\rangle=\left(\left.\right|^{1} \Sigma^{+}, 3,0\right\rangle\right)$ or $\left.|S\rangle\left(\left.\right|^{1} \Sigma^{+}, 3,2\right\rangle\right)$. The population of the other states of the basis set is not drawn.

which do not belong to the model. The filtering is mainly done because we use a truncated basis set so it seems more consistent to avoid frequencies lying in a domain out of the model to ensure convergence if the field was applied with a larger basis set. An experimental OCT working with the full space could probably find very different and efficient pathways. We apply a bandpass filter around the four vibrational frequencies retained in the model with a width of about $25 \%$ of the vibrational frequency to remove irrelevant frequencies. The filtering of these unrealistic frequencies notably decreases the fidelity to $30 \%$ (first trial field) and 53\% (STIRAP as trial field). The pulses are optimized again in ten new iterations and filtered along the same lines. Only four cycles are necessary in each case to reach a stable fidelity of $99.9 \%$. The Fourier transforms of the final fields are shown in the lower panels of Fig. 6. The filtering also allows to decrease the final intensity of the control field and to find new pathways to reach the objective. The maximum amplitude does not exceed $10^{7} \mathrm{~V} \mathrm{~m}^{-1}$ when the STIRAP solution is the zero-order field and $810^{7} \mathrm{~V} \mathrm{~m}^{-1}$ in the other case. One can see in panels (c) and (d) of Fig. 6 that after filtering, the mechanism involves transitions via the $v=1$ vibrational level. One also observes in panel (d) that the new mechanism finds the $\Omega_{S}$ frequency which is typical of the STIRAP sequence.

As could be expected, OCT leads to a lower robustness than the one obtained in a STIRAP process for both trial fields. This is due to the higher complexity of the spectrum and of the pulse envelop. We show in Fig. 7 the realization of the CNOT gate with the optimized pulse starting from the STIRAP sequence. Only the main active populations are drawn. One sees for instance that the populations in states $|00\rangle$ and $|10\rangle$ do not evolve smoothly at the end of the process. 


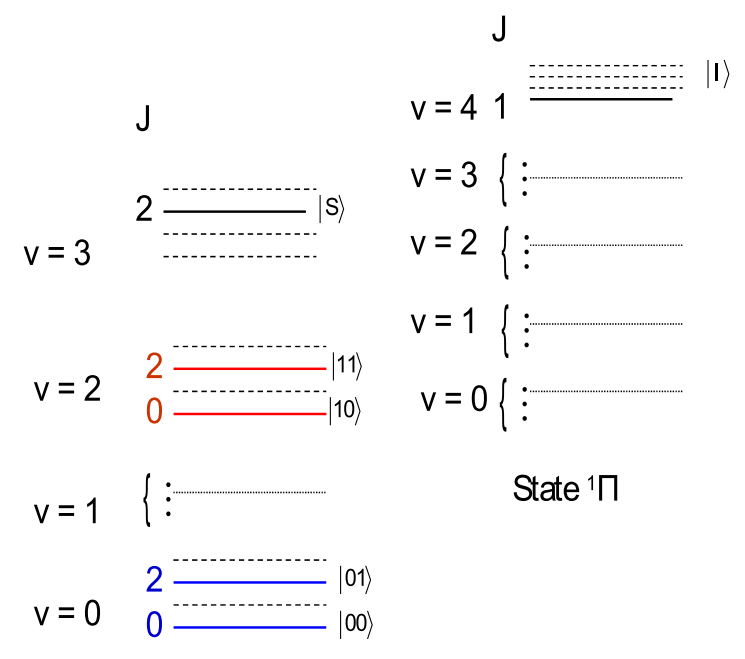

State ${ }^{1} \Sigma^{+}$

FIG. 8. (Color online) Scheme of the qubit states with $q_{c}$ in the vibration for the diatomic example. $v$ and $J$ are the vibrational and rotational quantum numbers, respectively. $|S\rangle$ and $|I\rangle$ are the shelving and intermediate states of the STIRAP sequence (see Fig. 1)

Shorter pulse durations have been considered. The structures of the trial fields are similar to those of the long pulses (1.4 ns) but the duration is now of $140 \mathrm{ps}$. The convergence of the algorithm is slower but a good fidelity is still achieved. However, when the maximum intensity of field is of the same order of magnitude $\left(10^{7}-7 \quad 10^{7} \mathrm{~V} \mathrm{~m}^{-1}\right)$, the spectrum is composed of a lot of unrealistic frequencies and the filtering procedure is now inefficient. It is possible to get a spectrum similar to the one of the long pulse but at the price of an amplitude at least 10 times larger. This confirms that long control duration has to be chosen even with OCT.

\section{B. Assignment 2: Control qubit encoded in the vibrational states}

This case is illustrated in Fig. 8. The STIRAP process also misses the objective with the assignment 2 . The expected transition is $|10\rangle\left(\left|{ }^{1} \Sigma^{+}, 2,0\right\rangle\right) \leftrightarrow|11\rangle\left(\left|{ }^{1} \Sigma^{+}, 2,2\right\rangle\right)$, i.e., a rotational inversion in a given vibrational state. This time, the $\Omega_{S} \Omega_{11} \Omega_{10} \Omega_{S}$ pulse sequence does not act on the $|00\rangle$ and $|01\rangle$ states as expected. However, the states $|10\rangle\left(\left|{ }^{1} \Sigma^{+}, 2,0\right\rangle\right)$ and $|11\rangle\left(\left|{ }^{1} \Sigma^{+}, 2,2\right\rangle\right)$ both interact with the same pulse. For example, when $\Omega_{S} \Omega_{11}$ is applied, $\left.|11\rangle\left(\left.\right|^{1} \Sigma^{+}, 2,2\right\rangle\right)$ is transferred to the shelving state $|S\rangle=\left|{ }^{1} \Sigma^{+}, 3,2\right\rangle$, but $|10\rangle$ $\left(\left|{ }^{1} \Sigma^{+}, 2,0\right\rangle\right)$ is also transferred to another intrusive shelving state $\left|S^{\prime}\right\rangle=\left|{ }^{1} \Sigma^{+}, 3,0\right\rangle$ via the same intermediate one $\left|I^{\prime}\right\rangle=\left|{ }^{1} \Pi, 4,1\right\rangle$, so we are not able to make a CNOT gate by the STIRAP sequence using this assignment. This could be done by OCT as in the case of assignment 1 .

\section{Assignment 3: Qubit states in four different vibrational levels}

Figure 9 schematizes this third assignment. The adiabatic approach optimized by GA leads to a very high fidelity of the order of $98.9 \%$ when the qubit states are encoded in rota-

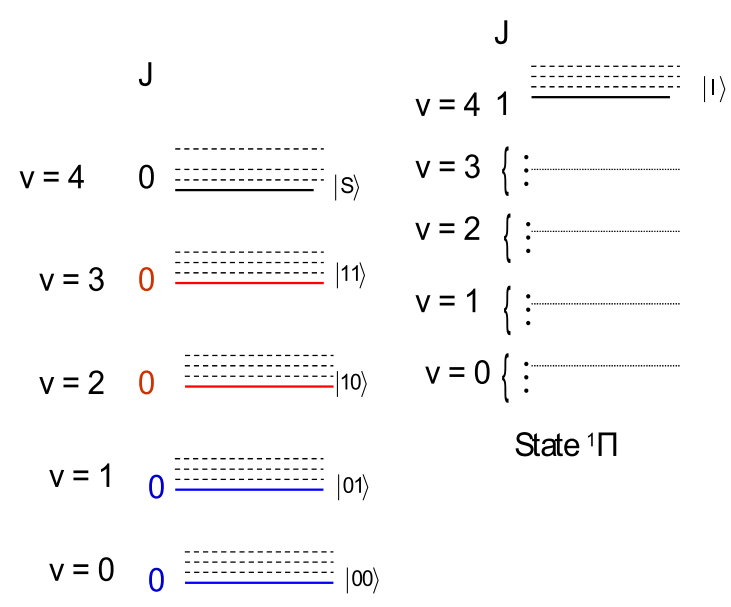

State ${ }^{1} \Sigma^{+}$

FIG. 9. (Color online) Scheme of the qubit states belonging to four different vibrational states for the diatomic example. $v$ and $J$ are the vibrational and rotational quantum numbers, respectively. $|S\rangle$ and $|I\rangle$ are the shelving and intermediate states of the STIRAP sequence (see Fig. 1)

tional states of four different vibrational states (assignment 3). The evolution of the populations is shown in Fig. 10 starting from a superposition of the four qubit states with unequal weights. The optimized parameters are given in Table A of Appendix B. We have observed that the phase constraint requires longer pulses than the classical population inversion. The optimized pulse is longer than in the previous examples. The width $\gamma_{k}=0.6$ ns of each pulse [Eq. (3)] is fixed by the rotational resolution (about $5.9 \times 10^{-2} \mathrm{~cm}^{-1}$ ) leading to a time scale of $0.56 \mathrm{~ns}$. We have checked that longer pulses do not correct the pathology of assignments

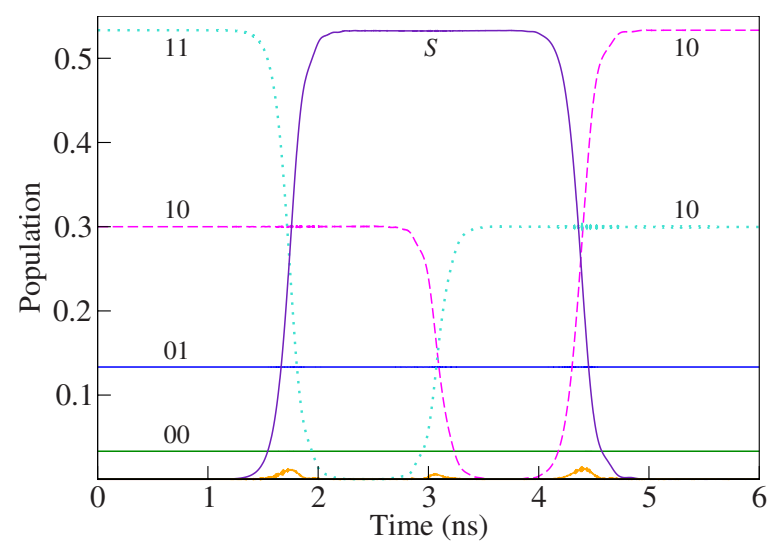

FIG. 10. (Color online) CNOT gate by the STIRAP sequence with phase constraint [Eq. (2)] when the qubit states are encoded in the rovibrational states $J=0$ of different vibrational states $v=0,1,2$, and 3 (see Fig. 9). The initial state is a superposition of all the qubit states with unequal weights. The populations in the states $|00\rangle$ $\left(\left|{ }^{1} \Sigma^{+}, 0,0\right\rangle\right)$ and $|01\rangle\left(\left|{ }^{1} \Sigma^{+}, 1,0\right\rangle\right)$ remain constant. One observes the exchange of the populations in $|11\rangle\left(\left|{ }^{1} \Sigma^{+}, 3,0\right\rangle\right)$ in dotted line and of $\left.|10\rangle\left(\left.\right|^{1} \Sigma^{+}, 2,0\right\rangle\right)$ in dashed line. The shelving state $|S\rangle$ is $\left(\left|{ }^{1} \Sigma^{+}, 4,0\right\rangle\right)$. 
TABLE I. State numbers, energies, and transition dipole moments of vibrational states extracted from Table I of Ref. [7] for the molecule $\mathrm{SCCl}_{2}$ and qubit assignment for the CNOT gate (see also Fig. 1).

\begin{tabular}{ccccc}
\hline $\begin{array}{c}\text { Vibrational } \\
\text { state } \\
\text { number } 7\end{array}$ & $\begin{array}{c}\text { Rotational } \\
\text { state }\end{array}$ & $\begin{array}{c}\text { Vibrational } \\
\text { wave number } \\
\left(\mathrm{cm}^{-1}\right)\end{array}$ & $\begin{array}{c}\text { Dipolar } \\
\text { moments } \\
\text { (D) }\end{array}$ & $\begin{array}{c}\text { Qubit } \\
\text { assignment }\end{array}$ \\
\hline$X$ state & & & & \\
11 & $\left|0_{00}\right\rangle$ & 8239.53 & 0.36 & $|00\rangle$ \\
12 & $\left|0_{00}\right\rangle$ & 8246.35 & 0.38 & $|01\rangle$ \\
13 & $\left|0_{00}\right\rangle$ & 8264.26 & 0.96 & $|10\rangle$ \\
14 & $\left|0_{00}\right\rangle$ & 8273.98 & 0.75 & $|11\rangle$ \\
27 & $\left|0_{00}\right\rangle$ & 8481.07 & 0.84 & $|S\rangle$ \\
& & & & $|I\rangle$ \\
\hline state & & & & \\
29 & $\left|1_{01}\right\rangle$ & 35125.0 & & \\
\hline \hline
\end{tabular}

1 and 2 which is not related to the rotational resolution but to the large detunings allowed by the STIRAP process.

\section{POLYATOMIC MOLECULE}

We finally show that the optimized adiabatic strategy can be efficient in a polyatomic molecule. We consider only the assignment 3 where the rotational states belong to different vibrational levels since the other assignments are irrelevant. We use a molecular system already proposed in the literature in the context of vibrational computing [7]. The qubit states and the shelving states are chosen among rovibrational states of the $X$ ground electronic surface of thiophosgene $\mathrm{SCCl}_{2}$. The intermediate state is a rovibrational state of the $B$ excited electronic state. We take back the vibrational levels and vibrational transition dipole moments given in Table I of Ref. [7]. Other vibrational levels which are not taken into account in the computation are expected to play a minor role in the control. Table I gathers the data extracted from Table I of Ref. [7] for the four qubit states, the shelving, and intermediate states. The states $(|10\rangle,|11\rangle,|S\rangle)$ which exchange their population in the STIRAP process to realize the CNOT gate are chosen among the contiguous vibrational levels with the largest transition dipole moments. The $\mathrm{SCCl}_{2}$ molecule is an asymmetric top molecule with rotational constants $A=0.119 \mathrm{~cm}^{-1}, B=0.116 \mathrm{~cm}^{-1}$, and $C=0.059 \mathrm{~cm}^{-1}$ in the $X$ state and $A=0.139 \mathrm{~cm}^{-1}, \quad B=0.084 \mathrm{~cm}^{-1}$, and $C=0.052 \mathrm{~cm}^{-1}$ in the $B$ state. The rotational factors of the transition dipole moment for the asymmetric top molecule are computed in the eigenbasis of the symmetric-top molecule (see Appendix A). The rotational states for each qubit state and the shelving state are, respectively, $J=0, K_{a}=0$, and $K_{c}=0$ and $J=1, K_{a}=0$, and $K_{c}=1$ for the intermediate state. The simulation includes $(29 \times 9)$ states, i.e., 28 vibrational states in the $X$ state and one vibrational state in the $B$ state with nine rotational states (up to $J=2$ ) per vibrational levels.

The optimized parameters [Eq. (3)] for the CNOT gate are given in Table B. We have observed that the APT sequence of the diatomic molecule is already an optimized field for the

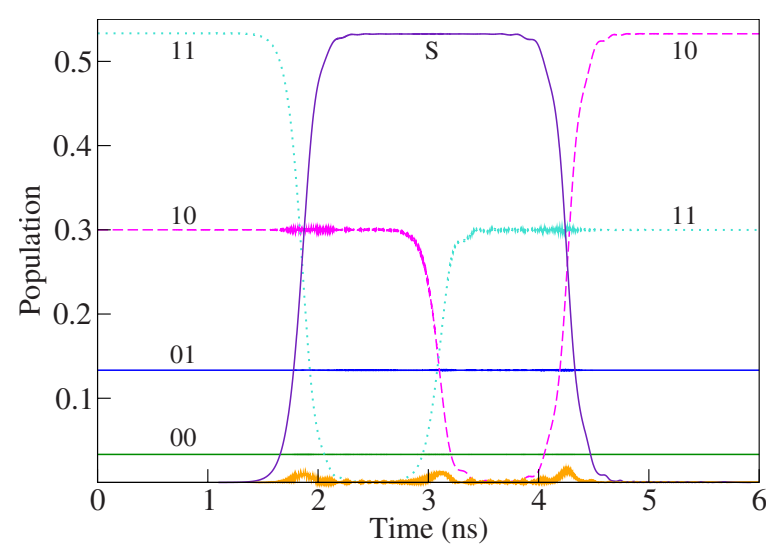

FIG. 11. (Color online) CNOT gate by the STIRAP sequence with phase constraint [Eq. (2)] and with the qubit states encoded in the rovibrational states $\left|0_{00}\right\rangle$ of different excited vibrational states of $\mathrm{SCCl}_{2}$ (see Table I). Evolution of the populations in the qubit states $|00\rangle$ and $|01\rangle$ which remain constant. Evolution of the interchanging populations $|10\rangle$ in dashed line and $|11\rangle$ in dotted line.

polyatomic system because the rotational resolution is of the same order (about $5.2 \times 10^{-2} \mathrm{~cm}^{-2}$ ). The fidelity is $99.1 \%$ (with a phase constraint, i.e., for a complete unitary transformation). Figure 11 shows the evolution of the population when the CNOT gate is applied to a superposition with different weights. Similar results are obtained for any superposition. The APT sequence is robust and the fidelity is very high but it is very difficult to decrease the duration of the optimized sequence. As already mentioned for the diatomic molecule, this remark indicates that a long control duration has to be chosen when the phase constraint is taken into account.

\section{CONCLUSION}

A promising architecture to implement scalable quantum computation is arrays of entities which can be individually addressed and controlled independently from the states of the other ones but ensure coupling among qubits encoded in neighboring sites. A challenging possibility is an ensemble of trapped polar molecules [10,35-37]. Formation and control of ultracold molecule are currently a domain in full expansion [44]. In this context, the realization of quantum networks requires to split the overall process in several intramolecular and intermolecular gates. Here, we have examined the implementation of a CNOT intramolecular gate. We have mainly analyzed the role of the assignment in rotational states when a CNOT gate is implemented by APT or OCT. APT operates in the ns range. It offers the advantage of the simplicity and robustness of control pulses but it is relevant only for a particular encoding scheme (scheme 3 ) which involves rovibrational states belonging to four different vibrational manifolds. Even very long control durations (some hundreds of nanoseconds) do not prevent unwanted transitions for the encodings 1 and 2. The impossibility of implementing the quantum gates in theses cases by APT is thus due to the encoding. In the favorable case (scheme 3), the phase constraint which is essential to realize a quantum unitary transformation is more difficult to satisfy than the simple 
population inversion. This constitutes an intrinsic limitation to quantum computing with respect to classical one when using optimized APT. In view of practical implementations in molecular systems, classical operations such as adder or subtractor could be therefore more promising in a near future.

OCT achieves a high fidelity of the quantum gate with phase constraint for any assignment of the qubit states. In any case, the adiabatic solution can be used as a very efficient trial field. Since there exists no unique optimal solution, we can select by an adequate choice of the trial field and by the use of spectral constraints a particular control field and a particular pathway for the dynamics. The APT starting guess is a trick inspired from a basic mechanism with a cycling in the uv-visible range through an excited electronic state. The advantages are a smaller intensity of the optimal field and a simpler structure but obviously OCT can work anyhow with different trial fields and shorter durations. However, we note that the third typical frequency of the STIRAP sequence has been recovered by OCT starting with a different trial field based on two frequencies only. No limitation on the control duration has been observed with OCT but the structure of the optimal control field can be more complicated for a short duration. There is no physical reason to select a simple control field rather than a more complex one. However, to our knowledge, a control field with a simple structure in the time or frequency domain is generally easier to implement experimentally and more robust with respect to experimental inaccuracies or with respect to inaccuracies of the theoretical model. Finally, we remark that the present approach based on a cycling between the ground and an excited electronic state is a strategy complementary to the previous study done in midinfrared domain [8].

\section{ACKNOWLEDGMENTS}

The computing facilities of IDRIS (Projects No. 061247 and No. 2006 0811429) as well the financial support of the FNRS in the University of Liège SUN Nic2 project are gratefully acknowledged. We thank for the support of the COST Action CM0702 CUSPFEL. The research has also been supported by Triangle de la Physique Convention No. 2009-039T.

\section{APPENDIX A: ROTATIONAL FACTOR OF THE TRANSITION MOMENT}

We first discuss the diatomic case. By using the transformation of the electric-dipole moment operator from the body fixed to the space-fixed frame $\mu_{p}=\sum_{q=-1}^{1} D_{p q}^{(1) *}(\phi, \theta, 0) \mu_{q}$, where the tensorial components $p$ and $q$ refer to the laboratory frame and the molecular one, respectively, the matrix element of the dipolar interaction between two rovibronic states $|k\rangle=\left|\Lambda_{k}, v_{k}, J_{k} M_{k} \Omega_{k}\right\rangle$ becomes

$$
\begin{aligned}
\vec{\mu}_{i f} \cdot \vec{E}= & \sum_{p, q=0, \pm 1}(-1)^{q} E_{-p}\left\langle\chi_{v_{f}}\left|\mu_{q}^{\Lambda_{f} \Lambda_{i}}\right| \chi_{v_{i}}\right\rangle \\
& \times\left\langle J_{i} M_{i} \Omega_{i}\left|D_{p q}^{(1) *}\right| J_{f} M_{f} \Omega_{f}\right\rangle,
\end{aligned}
$$

where $|J M \Omega\rangle=(-1)^{M-\Omega} \sqrt{\frac{2 J+1}{4 \pi}} D_{M \Omega}^{(J)}(\theta, \phi, 0)$ is the Wigner ro- tation matrix for a diatomic system, with $J$ the total angular momentum, $M$ its projection on the OZ laboratory axis, $\Omega$ its projection on the $\mathrm{Oz}$ molecular axis, and $\chi_{v}$ are the vibrational functions. The rotational factors of the transition elements are expressed in terms of the Wigner $3 j$ symbols [45]

$$
\begin{aligned}
\left\langle J_{i} M_{i} \Omega_{i}\left|D_{p q}^{(1) *}\right| J_{f} M_{f} \Omega_{f}\right\rangle= & \sqrt{\left(2 J_{i}+1\right)\left(2 J_{f}+1\right)}\left(\begin{array}{ccc}
J_{i} & 1 & J_{f} \\
-M_{i} & p & M_{f}
\end{array}\right) \\
& \times\left(\begin{array}{ccc}
J_{i} & 1 & J_{f} \\
-\Omega_{i} & q & \Omega_{f}
\end{array}\right),
\end{aligned}
$$

with

$$
\begin{aligned}
\left(\begin{array}{ccc}
j_{1} & j_{2} & j_{3} \\
m_{1} & m_{2} & m_{3}
\end{array}\right)= & (-1)^{j_{1}-j_{2}-m_{3}} \frac{1}{\sqrt{2 j_{3}+1}} \\
& \times\left\langle j_{1} m_{1} j_{2} m_{2} \mid j_{1} j_{2} j_{3},-m_{3}\right\rangle .
\end{aligned}
$$

We consider a linearly polarized electric field leading to $p=0$ and a perpendicular transition between $\Sigma$ and $\Pi$ states so that $q=1$.

The rotational Hamiltonian of the asymmetric top is

$$
\begin{aligned}
H_{r o t}= & \frac{1}{h^{2}}\left(A \hat{J}_{a}^{2}+B \hat{J}_{b}^{2}+C \hat{J}_{c}^{2}\right) \\
= & \frac{1}{h^{2}}\left\{\frac{B+C}{2} \hat{J}^{2}+\left(A-\frac{B+C}{2}\right) \hat{J}_{z}^{2}\right. \\
& \left.+\frac{B-C}{4}\left[\left(\hat{J}_{z}^{+}\right)^{2}+\left(\hat{J}_{z}^{-}\right)^{2}\right]\right\},
\end{aligned}
$$

where $A, B$, and $C$ are the rotational constants. The energies $E_{J_{K_{a}} K_{c}}$ for $J=0,1,2$ are calculated by diagonalizing the rotational Hamiltonian in the basis set of eigenvectors $|J M K\rangle$ of the symmetric top. Eigenvectors and eigenvalues are tabulated in Ref. [45]. The rotational factors $\left\langle J_{K_{a}^{\prime} K_{c}^{\prime}}^{\prime}\left|D_{p q}\right| J_{K_{a}^{\prime \prime} K_{c}^{\prime \prime}}^{\prime \prime}\right\rangle$ are the corresponding combinations of the rotational factors of the symmetrical top

$$
\begin{aligned}
\left\langle J_{i} M_{i} K_{i}\left|D_{p q}\right| J_{f} M_{f} K_{f}\right\rangle= & \sqrt{\left(2 J_{i}+1\right)\left(2 J_{f}+1\right)}\left(\begin{array}{ccc}
J_{i} & 1 & J_{f} \\
-M_{i} & p & M_{f}
\end{array}\right) \\
& \times\left(\begin{array}{ccc}
J_{i} & 1 & J_{f} \\
-K_{i} & q & K_{f}
\end{array}\right) .
\end{aligned}
$$

We consider here the cases with $p=0$ (linear polarization) and $q=0$ (parallel transition $\mu_{a} \neq 0$ ). 


\section{APPENDIX B: PARAMETERS OF THE PULSES}

Optimized parameters of the STIRAP sequence $\Omega_{S} \Omega_{11} \Omega_{10} \Omega_{S}$ (see Fig. 1) implemented in the NaCs system for the three assignments presented in Sec. III. The parameters are those of Eq. (3).

\begin{tabular}{cccccccrrrr} 
& $\begin{array}{c}\gamma_{k=1,4} \\
(\mathrm{~ns})\end{array}$ & $\begin{array}{c}E_{01} \\
(\mathrm{~V} / \mathrm{m})\end{array}$ & $\begin{array}{c}t_{1} \\
(\mathrm{~ns})\end{array}$ & $\begin{array}{c}E_{02} \\
(\mathrm{~V} / \mathrm{m})\end{array}$ & $\begin{array}{c}t_{2} \\
(\mathrm{~ns})\end{array}$ & $\begin{array}{c}E_{03} \\
(\mathrm{~V} / \mathrm{m})\end{array}$ & $\begin{array}{c}t_{3} \\
(\mathrm{~ns})\end{array}$ & $\begin{array}{c}E_{04} \\
(\mathrm{~V} / \mathrm{m})\end{array}$ \\
\hline \hline 1 & 0.145 & 13.16 & $10^{6}$ & 0.363 & $-7.3010^{6}$ & 0.610 & $5.6610^{6}$ & 0.815 & $-10.1810^{6}$ \\
2 & 0.145 & -13.52 & $10^{6}$ & 0.363 & $20.0510^{6}$ & 0.597 & $18.9710^{6}$ & 0.837 & $14.2410^{6}$ & 1.038 \\
3 & 0.605 & 4.52 & $10^{6}$ & 1.209 & $-4.061 \quad 10^{6}$ & 2.430 & $2.78810^{6}$ & 3.702 & $-4.02010^{6}$ & 4.892 \\
\hline
\end{tabular}

Optimized parameters of the STIRAP sequence $\Omega_{S} \Omega_{11} \Omega_{10} \Omega_{S}$ (see Fig. 1) implemented in the $\mathrm{SCCl}_{2}$ system for assignment in four different vibrational states. The parameters are those of Eq. (3).

\begin{tabular}{ccccccccc}
$\begin{array}{c}\gamma_{k=1,4} \\
(\mathrm{~ns})\end{array}$ & $\begin{array}{c}E_{01} \\
(\mathrm{~V} / \mathrm{m})\end{array}$ & $\begin{array}{c}t_{1} \\
(\mathrm{~ns})\end{array}$ & $\begin{array}{c}E_{02} \\
(\mathrm{~V} / \mathrm{m})\end{array}$ & $\begin{array}{c}t_{2} \\
(\mathrm{~ns})\end{array}$ & $\begin{array}{c}E_{03} \\
(\mathrm{~V} / \mathrm{m})\end{array}$ & $\begin{array}{c}t_{3} \\
(\mathrm{~ns})\end{array}$ & $\begin{array}{c}E_{04} \\
(\mathrm{~V} / \mathrm{m})\end{array}$ \\
\hline \hline 0.605 & $4.520 \quad 10^{6}$ & 1.209 & $-4.061 \quad 10^{6}$ & 2.430 & $2.78810^{6}$ & 3.702 & $-4.02010^{6}$ & 4.892 \\
\hline
\end{tabular}

[1] F. Remacle and R. D. Levine, Phys. Rev. A 73, 033820 (2006).

[2] L. Bomble, B. Lavorel, F. Remacle, and M. Desouter-Lecomte, J. Chem. Phys. 128, 194308 (2008).

[3] C. M. Tesch and R. de Vivie-Riedle, J. Chem. Phys. 121, 12158 (2004).

[4] B. M. R. Korff, U. Troppmann, K. L. Kompa, and R. de VivieRiedle, J. Chem. Phys. 123, 244509 (2005).

[5] S. Suzuki, K. Mishima, and K. Yamashita, Chem. Phys. Lett. 410, 358 (2005).

[6] M. Zhao and D. Babikov, J. Chem. Phys. 126, 204102 (2007).

[7] D. Weidinger and M. Gruebele, Mol. Phys. 105, 1999 (2007).

[8] M. Tsubouchi and T. Momose, Phys. Rev. A 77, 052326 (2008).

[9] D. DeMille, Phys. Rev. Lett. 88, 067901 (2002).

[10] E. Kuznetsova, R. Côté, K. Kirby, and S. F. Yelin, Phys. Rev. A 78, 012313 (2008).

[11] J. Vala, Z. Amitay, B. Zhang, S. R. Leone, and R. Kosloff, Phys. Rev. A 66, 062316 (2002).

[12] W. Zhu, J. Bottina, and H. Rabitz, J. Chem. Phys. 108, 1953 (1998); W. Zhu and H. Rabitz, ibid. 109, 385 (1998).

[13] Y. Ohtsuki, Y. Teranishi, P. Saalfrank, G. Turinici, and H. Rabitz, Phys. Rev. A 75, 033407 (2007).

[14] D. E. Goldberg, Genetic Algorithms in Search Optimization and Machine Learning (Addison-Wesley, Reading, MA, 2005).

[15] T. Cheng and A. Brow, J. Chem. Phys. 124, 034111 (2006).

[16] D. Sugny, C. Kontz, M. Ndong, Y. Justum, G. Dive, and M. Desouter-Lecomte, Phys. Rev. A 74, 043419 (2006).

[17] D. Sugny, M. Ndong, D. Lauvergnat, Y. Justum, and M. Desouter-Lecomte, J. Photochem. Photobiol., A 190, 359 (2007).

[18] M. Ndong, D. Lauvergnat, X. Chapuisat, and M. DesouterLecomte, J. Chem. Phys. 126, 244505 (2007).

[19] M. Schröder and A. Brown, J. Chem. Phys. 131, 034101 (2009).

[20] K. Shioya, K. Mishima, and K. Yamashita, Mol. Phys. 105,
1283 (2007).

[21] L. Bomble, D. Lauvergnat, F. Remacle, and M. DesouterLecomte, J. Chem. Phys. 128, 064110 (2008).

[22] L. Bomble, D. Lauvergnat, F. Remacle, and M. DesouterLecomte, Phys. Rev. A 80, 022332 (2009).

[23] Y. Ohtsuki, Chem. Phys. Lett. 404, 126 (2005).

[24] K. Mishima, K. Tokumo, and K. Yamashita, Chem. Phys. 343, 61 (2008); K. Mishima and K. Yamashita, ibid. 361, 106 (2009).

[25] N. V. Vitanov, T. Halfmann, B. Shore, and K. Bergmann, Annu. Rev. Phys. Chem. 52, 763 (2001).

[26] Z. Kis and F. Renzoni, Phys. Rev. A 65, 032318 (2002).

[27] H. Goto and K. Ichimura, Phys. Rev. A 70, 012305 (2004).

[28] D. Daems, S. Guérin, and N. J. Cerf, Phys. Rev. A 78, 042322 (2008).

[29] C. Menzel-Jones and M. Shapiro, Phys. Rev. A 75, 052308 (2007).

[30] V. Kurkal and S. A. Rice, Chem. Phys. Lett. 344, 125 (2001).

[31] I. Vrabel and W. Jakubetz, J. Chem. Phys. 118, 7366 (2003).

[32] C. Gollub and R. de Vivie-Riedle, Phys. Rev. A 78, 033424 (2008).

[33] J. Deiglmayr, A. Grochola, M. Repp, K. Mörtlbauer, C. Glück, J. Lange, O. Dulieu, R. Wester, and M. Weidemüller, Phys. Rev. Lett. 101, 133004 (2008).

[34] M. Viteau, A. Chotia, M. Allegrini, N. Bouloufa, O. Dulieu, D. Comparat, and P. Pillet, Science 321, 232 (2008).

[35] K. Mishima and K. Yamashita, J. Chem. Phys. 130, 034108 (2009).

[36] E. Charron, P. Milman, A. Keller, and O. Atabek, Phys. Rev. A 75, 033414 (2007); 77, 039907E (2008).

[37] L. D. Carr, D. Mille, R. V. Krems, and J. Ye, New J. Phys. 11, 055049 (2009).

[38] W. H. Press, B. P. Flannery, S. A. Teukolsky, and W. T. Vetterling, Numerical Recipes (Cambridge University Press, Cambridge, England, 1986).

[39] M. Zhao and D. Babikov, J. Chem. Phys. 125, 024105 (2006). 
[40] The code used is taken from http://eodev.sourceforge.net/

[41] J. P. Palao and R. Kosloff, Phys. Rev. A 68, 062308 (2003).

[42] C. Gollub, M. Kowalewski, and R. de Vivie-Riedle, Phys. Rev. Lett. 101, 073002 (2008); M. Lapert, R. Tehini, G. Turinici, and D. Sugny, Phys. Rev. A 79, 063411 (2009).
[43] M. Aymar and O. Dulieu, Mol. Phys. 105, 1733 (2007).

[44] O. Dulieu and C. Gabbanini, Rep. Prog. Phys. 72, 086401 (2009).

[45] R. N. Zare, Angular Momentum: Understanding Spatial Aspects in Chemistry and Physics (Wiley, New York, 1988). 